\title{
An Energy-Efficient 1.2V 4-Channel Wireless CMOS Potentiostat for Amperometric Biosensors
}

\author{
Virgilio Valente, Matthew Schormans and Andreas Demosthenous \\ Department of Electronic and Electrical Engineering, \\ University College London, WC1E 7JE, London, United Kingdom. \\ Contact Email: \{v.valente, matthew.schormans.10, a.demosthenous\}@ucl.ac.uk
}

\begin{abstract}
Point-of-care (PoC) diagnostics rely on the design of low-power and miniaturized readout units that can offer rapid and accurate test results, replacing the need for specialized equipment. CMOS technology can be exploited in order to design complex systems while achieving high energy efficiency for suitable operation in a mobile settings. This paper presents the design of a novel energy-efficient 4-channel wireless potentiostat chip, based on a dual-slope ADC architecture, that features a low-complexity wireless unit and a calibration approach that does not require additional circuitry. The chip was designed in a $0.35 \mu \mathrm{m}$ CMOS process. The simulated results suggest that each potentiostat channel can achieve an estimated energy efficiency of $2.5 \mathrm{pJ} / \mathrm{bit}$ from a $1.2 \mathrm{~V}$ supply.
\end{abstract}

\section{INTRODUCTION}

Point-of-care (PoC) diagnostics promise to improve the management of infectious diseases by offering rapid and accurate test results, especially in low-resource settings, allowing for a timely initiation of appropriate interventions [1]. The development of handheld platforms with functionality comparable to laboratory-based equipment is a key aspect to enable widespread adoption of PoC devices.

Electrochemical sensing is an attractive method to provide label-free, real-time quantitative detection and monitoring of target analytes. Electrochemical methods rely on the use of a feedback circuit, a potentiostat, which regulates a current flowing between a working and a counter electrode (WE and $\mathrm{CE}$ ) in order to keep a desired potential between the WE and a reference electrode (RE).

CMOS electrochemical platforms have been developed for numerous applications, including biosensors for neurotransmitter monitoring [2] and air quality control [3]. In order to obtain high energy efficiency, current-mode dual-slope or $\Sigma \Delta$ ADC architecture have been successfully adopted [3] to digitize the sensor current as early as possible, thus avoiding power hungry signal conditioning and amplification stages and achieve power consumptions in the order of tens to few hundreds of $\mu \mathrm{W} /$ channel. This paper presents the design and operation of a novel energy-efficient wireless potentiostat architecture based on a dual-slope ADC architecture, with 4 independently configurable channels. Simulated results show that each potentiostat channel can

This research is supported by the UK Engineering and Physical Sciences Research Council (EPSRC) under project i-sense (www.i-sense.org.uk).

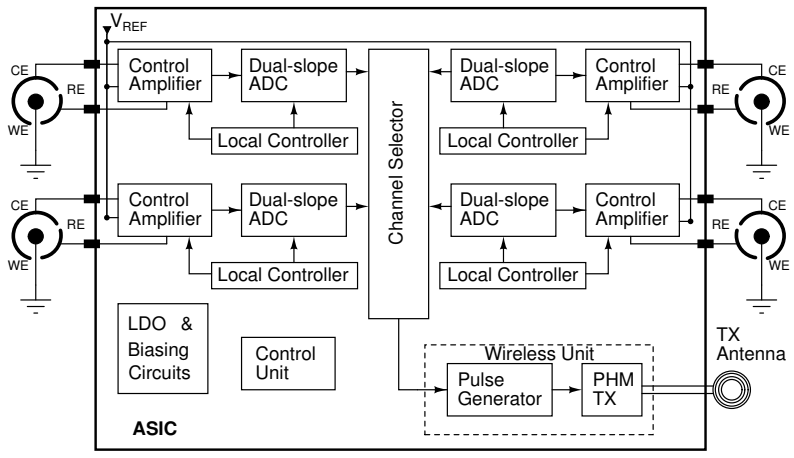

Fig. 1: Architecture of the 4-channel wireless potentiostat.

achieve an energy efficiency in the order of $\mathrm{pJ} / \mathrm{bit}$. The ADC is input-modulated in order to extend its dynamic range between $\mathrm{pA}$ to $\mu \mathrm{A}$ and features a novel wireless transmission strategy that greatly reduces the complexity of the wireless unit.

\section{Potentiostat Channel Design}

The architecture of the 4-channel wireless potentiostat system is shown in Fig. 1. Each potentiostat channel consists of a dual-mode control amplifier, a dual-slope ADC and a local controller. The wireless unit can be connected to each channel via an off-chip channel selector.

Conventional dual-slope ADCs, however, rely on the use of an on-chip counter and a fast clock, to determine the discharge time of the integrator capacitor, as shown in Fig. 2(a) If a wireless unit is then employed, the counter output needs to be encoded into a data stream via a modulation scheme and sent over a wireless link.

The proposed strategy is based on a modified pulse-harmonic modulation (PHM) scheme [4], whereby only two pulses are sent via the wireless link, one at the start and one at the end of the capacitor discharge period, as shown in Fig. 2(b). These pulses inherently encode the capacitor discharge time, which can be determined at the receiver end. This approach greatly reduces the complexity and power requirements of the wireless link. A pulse generator (PG) generates two short pulses and drive the PHM transmitter (TX) and the antenna. The detailed description of the wireless unit is found in [4]. 


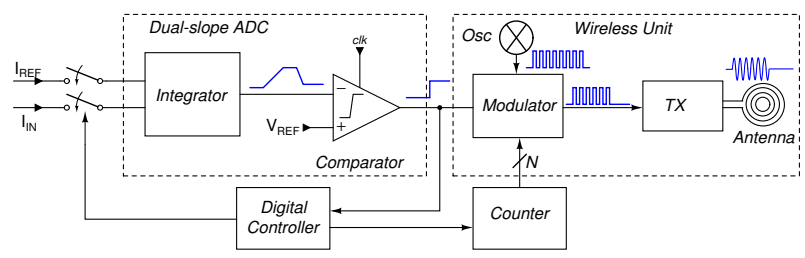

(a)

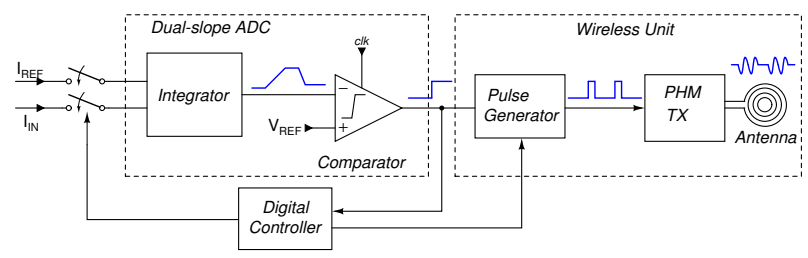

(b)

Fig. 2: Wireless potentiostat architecture. (a) Conventional and (b) proposed

A schematic of the channel is shown in Fig. 3. A dualmode current-mirror-based control amplifier is used to set the electrochemical cell potential $V_{R E-W E}$ to a fixed value. The control amplifier can set a positive $\mathrm{V}_{\mathrm{RE}} \mathrm{WE}$ or negative $\mathrm{V}_{\mathrm{RE}-\mathrm{WE}}$, depending on whether a reduction or oxidation current is measured during the chemical reaction. In the former case the working electrode (WE) is connected to ground, whereas in the latter case WE is connected to the supply potential $\mathrm{V}_{\mathrm{DD}}$.

The ADC is input-modulated by switches $S_{\text {modp }}$ and $S_{\text {modn }}$ in order to extend its dynamic range. The core of the dual-slope ADC consists of a switched-capacitor integrator featuring correlated-double sampling and a clocked comparator. The operation of the potentiostat channel can be described with the aid of the timing diagram in Fig. 3. After a global reset phase, $\phi_{\text {rst }}$, the output of the integrator, $V_{\text {int }}$ is held at $V_{\mathrm{REF}}$. During the integration phase, $\phi_{1}$, the input current, $\mathrm{I}_{\mathrm{IN}}$, is integrated on the capacitor $\mathrm{C}_{\mathrm{i}}$ for a fixed time, $t_{1}$ by closing switch $S_{1}$. During $t_{1}, \mathrm{~V}_{\text {int }}$ increases or decreases from $\mathrm{V}_{\mathrm{REF}}$, depending on the direction of $\mathrm{I}_{\mathrm{IN}}$. During the discharge phase, $\phi_{2}$, the reference DAC is enabled forcing $V_{\text {int }}$ to decrease (increase) toward $\mathrm{V}_{\mathrm{REF}}$. As the threshold is crossed the output of the comparator, $\mathrm{V}_{\text {comp }}$ toggles and the conversion is completed. The amplitude of the input current can be determined as:

$$
I_{I N}=\alpha \cdot k \cdot \frac{t_{2}}{t_{1}} \cdot I_{p}
$$

where $k$ is the input current modulation index equal to $t_{1} / T_{\text {mod }}$ and $\alpha$ is a gain factor due circuit non-idealities and $\mathrm{I}_{\mathrm{p}}$ is the programmable current from the reference DAC.

\section{A. Integrator}

The sensor current is integrated directly onto a $6.8 \mathrm{pF}$ capacitor, $\mathrm{V}_{\mathrm{i}}$. The choice of the capacitance value implies relatively long conversion times but results in low thermal noise performance. Correlated double sampling (CDS) is implemented by capacitor $\mathrm{C}_{\mathrm{S}}$, which stores the difference between the output amplifier and a reference voltage $V_{R E F}$ and biases amplifier $\mathrm{A}_{2}$.

The integrator opamp, $\mathrm{A}_{2}$, was implemented by the inverterbased class-AB amplifier shown in Fig. 4, together with the biasing circuit. An inverter-based architecture was chosen for high-efficiency, rail-to-rail output and low-voltage operation. The simulated performance of the opamp resulted in a low frequency gain of $89 \mathrm{~dB}$ and a bandwidth of $27 \mathrm{MHz}(100$ fF load) at a power supply of $1.2 \mathrm{~V}$. The simulated quiescent current was $680 \mathrm{nA}$ with a total current consumption of $920 \mathrm{nA}$. This results in a figure-of-merit (FOM) of 2930 $\mathrm{MHz} \cdot \mathrm{pF} / \mathrm{mA}$. The simulated input-referred RMS noise was 7.7 $\mu \mathrm{V}$ in a bandwidth between $10 \mathrm{~Hz}$ and $20 \mathrm{kHz}$.

\section{B. Comparator}

A clocked-comparator was adopted for low power consumption. The schematic of the comparator is shown in Fig. 5 It comprises an input latched stage $\left(\mathrm{M}_{\mathrm{N} 1-4}\right.$ and $\left.\mathrm{M}_{\mathrm{P} 1-6}\right)$, followed by an SR-latch $\left(\mathrm{M}_{\mathrm{N} 5-8}\right.$ and $\left.\mathrm{M}_{\mathrm{P} 7-10}\right)$ and output buffers. In order to reduce the comparator offset, the load capacitance of the input stage, $\mathrm{C}_{\mathrm{OS}}$, was implemented by a 6-bit capacitor array. The layout of the capacitor array is shown in Fig. 6. Custom unit capacitors have been designed with minimum sizes to minimize the area of the array. The extracted capacitance of the unit capacitor is approximately $630 \mathrm{aF}$, resulting in a total $\mathrm{C}_{\mathrm{OS}}$ of approximately $40 \mathrm{fF}$. Postlayout simulations have shown that this value accommodates comparator offsets up to $20 \mathrm{mV}$.

\section{CHIP OPERATION}

The chip was designed in a $0.35 \mu \mathrm{m}$ 4-metal CMOS technology. It consists of 84 pads for a total area of 2.8 -by- $2.8 \mathrm{~mm}^{2}$. The chip layout is shown in Fig. 7 together with the layout of a pixel in the inset of the figure. The performance of the chip was simulated in Cadence Spectre. The key performance parameters are summarized in Table $\mathrm{I}$

TABLE I: Summary of Simulated Performance.

\begin{tabular}{ccc} 
PARAMETER & UNITS & VALUE \\
\hline \hline Technology & - & $0.35 \mu \mathrm{m} \mathrm{CMOS}$ \\
Number of pads & - & 84 \\
Chip area (incl.pads) & $\mathrm{mm}^{2}$ & 7.84 \\
Supply voltage & $\mathrm{V}$ & 1.2 \\
Number of channels & - & 4 \\
Channel area & $\mu \mathrm{m}^{2}$ & $670 \times 200$ \\
$\mathrm{P}_{\text {conv }} /$ ch @ 20 kHz & $\mu \mathrm{W}$ & 12.06 \\
Resolution & bits & $12-16$ \\
\hline
\end{tabular}

\section{A. Conversion}

Fig. 8 shows the transient operation of the potentiostat channel during one conversion. The ADC clock was set to $10 \mathrm{MHz}$ and the integration time to $409.6 \mu \mathrm{s}$, resulting in a resolution of 12 bits. A $1 \mu \mathrm{A}$ cell current $\mathrm{I}_{\text {CELL was modulated }}$ with an index $k$ of 100 resulting in an average input current, $\mathrm{I}_{\mathrm{IN}}$ of $10 \mathrm{nA}$. This current is integrated on the capacitor $C_{i}$ 

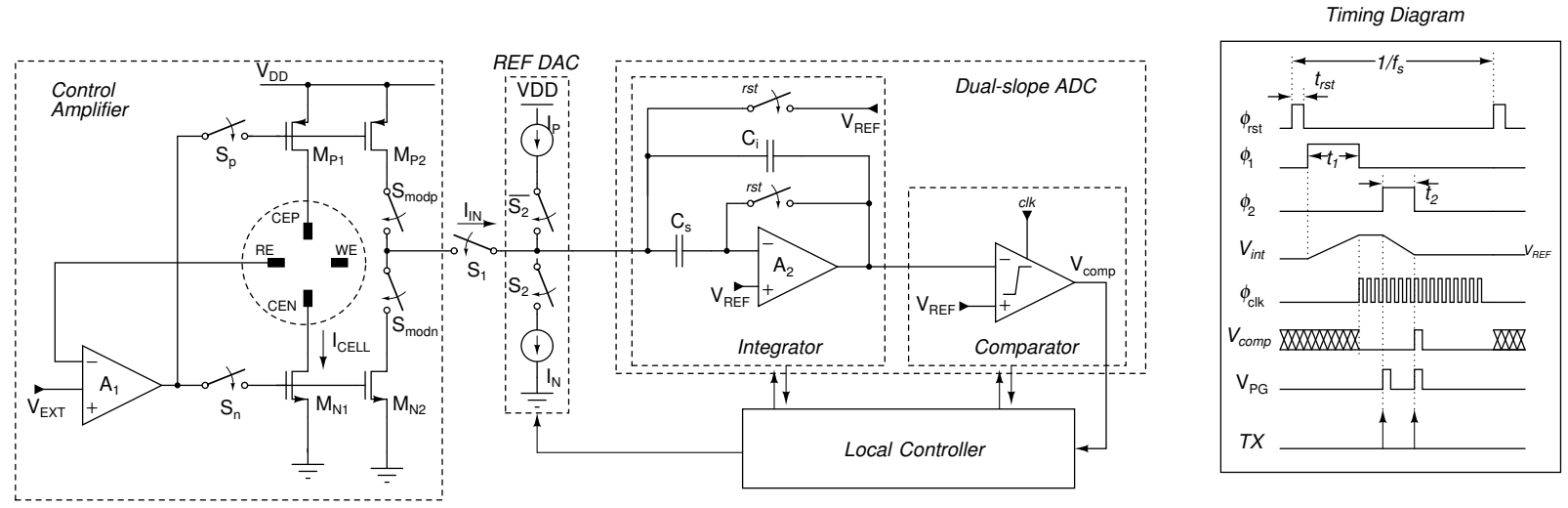

Fig. 3: Detailed schematic of a potentiostat channel.

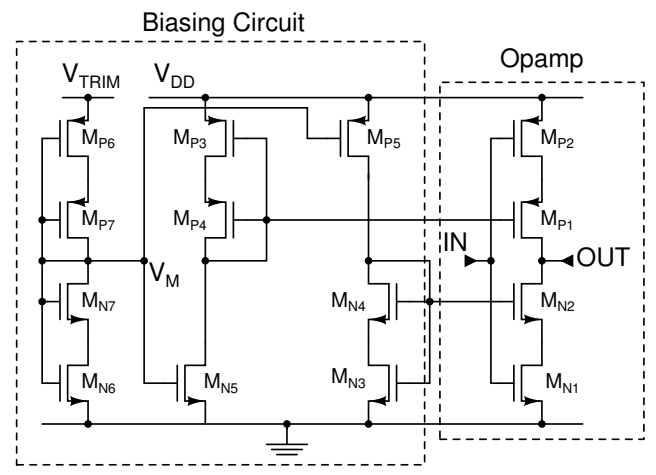

Fig. 4: Implementation of the integrator inverter-based opamp $\left(A_{2}\right.$ in Fig. 3) with biasing circuit.

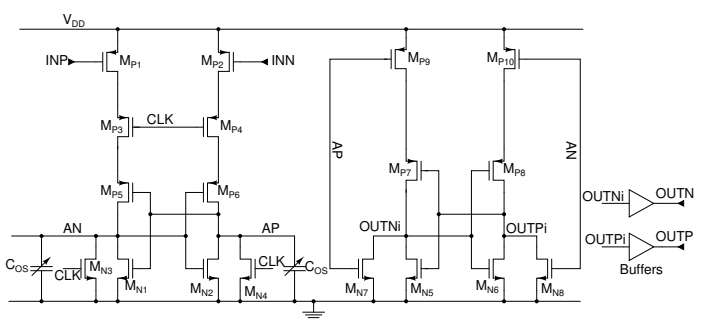

Fig. 5: Schematic of the clocked comparator with trimming capacitors $C_{\mathrm{OS}}$ for offset compensation.

resulting in $\mathrm{V}_{\text {int }}$ ramping up toward the supply voltage. After the end of the integration phase, $\mathrm{C}_{\mathrm{i}}$ is discharged back to the reference voltage by a $10 \mathrm{nA}$ (nominal) DAC current. The overall conversion takes approximately $0.8 \mathrm{~ms}$ and consumes $12.06 \mu \mathrm{W}$ resulting in an energy consumption of $2.35 \mathrm{pJ} / \mathrm{bit}$.

\section{B. Calibration}

A calibration step is needed in order to derive a gain factor, $\alpha$, in Eq. 1. which is mainly associated with variations in the nominal values of the REF DAC current, and to remove offset and non-idealities of the comparator. In the former case, a known current can be applied to the ADC and varied until $t_{1}$ equals $k \cdot t_{2}$ and hence the reference DAC current, $\mathrm{I}_{\mathrm{p}}$, equals the externally applied current. In the latter case, offset calibration

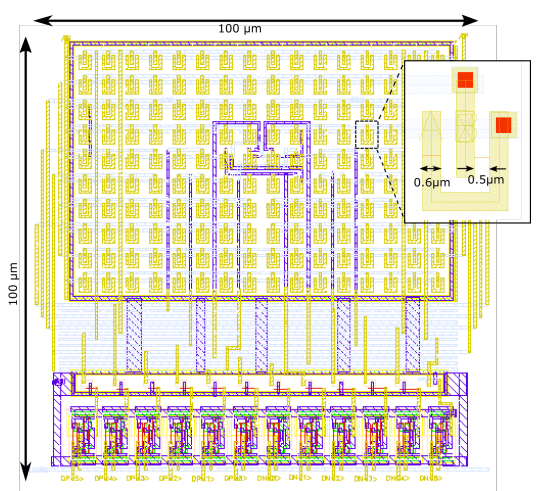

Fig. 6: Layout of implementation of $\mathrm{C}_{\mathrm{OS}}$.

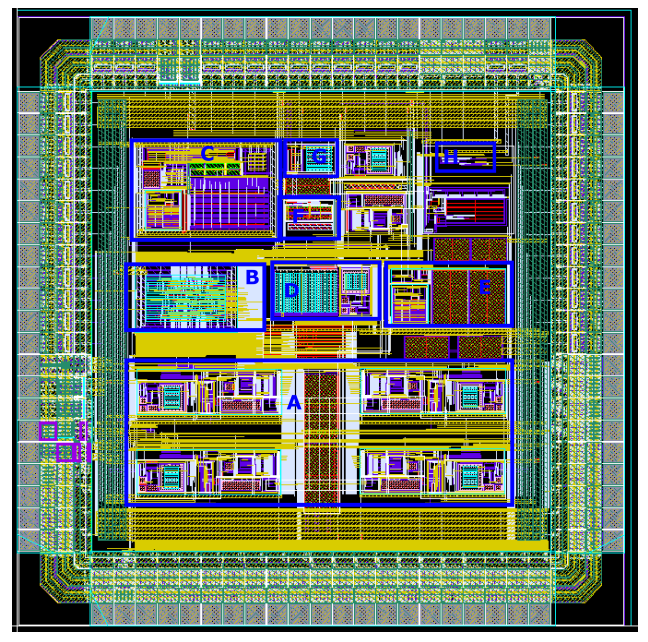

Fig. 7: Chip layout. A.4-channel dual-slope ADC with control amplifier B. 8-register SPI. C. Bandgap. D. LDO. E Bias cell. F. POR. G. Beta multiplier. H. TX. Unlabeled blocks represent test structures.

can be performed at system level, as illustrated by the transient simulations in Fig. 9 .

The operation is similar to that of a first order sigma-delta modulator. The reference DAC current is integrated until the integrator output, $\mathrm{V}_{\text {int }}$ crosses $\mathrm{V}_{\mathrm{REF}}+\mathrm{V}_{\mathrm{OS}}$, at which point the comparator toggles and the reference DAC current changes 

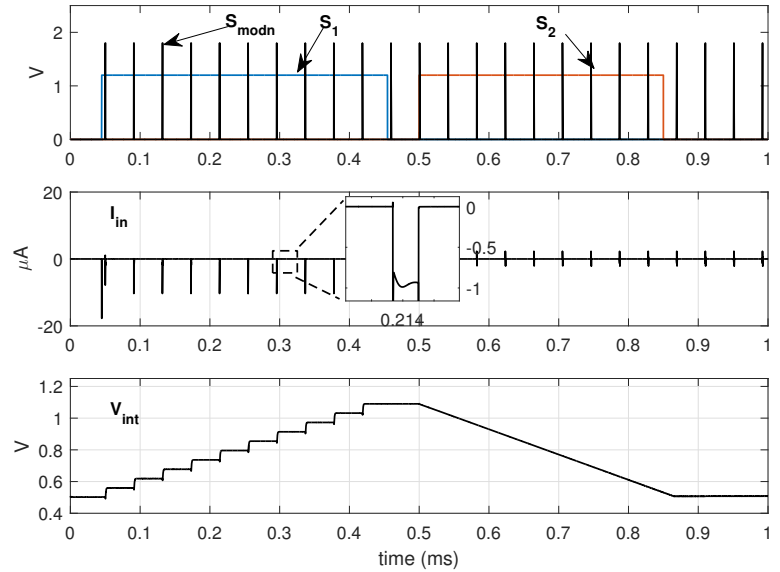

Fig. 8: Transient simulation of potentiostat channel operation.
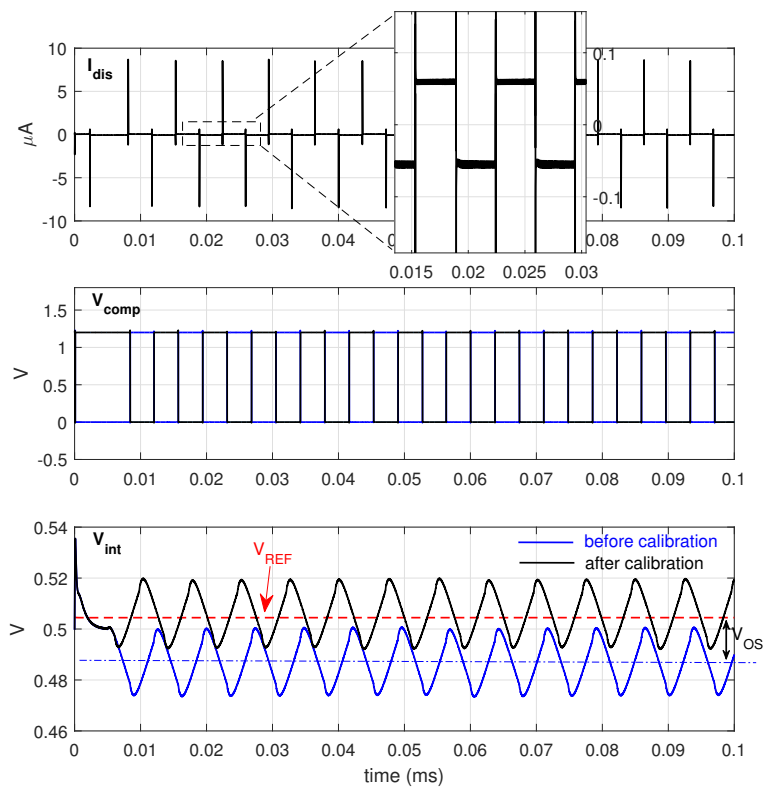

Fig. 9: Transient simulation of calibration of the potentiostat channel.

direction. The offset voltage, $\mathrm{V}_{\mathrm{OS}}$ can be determined by measuring the average of $\mathrm{V}_{\mathrm{int}}$ (with a simple first-order external RC filter). The offset can then be reduced by digitally trimming the offset capacitors $\mathrm{C}_{\mathrm{OS}}$ as described in the previous section. Simulation results have shown that the overall system offset can be reduced to a nominal value of $156 \mu \mathrm{V}$.

\section{Wireless transmission}

Fig. 10 shows the operation of the wireless unit. The PG generates two UWB-like pulses, one at the start of the conversion period, $\mathrm{T}_{\text {conv }}$ and one at the end of this period, when $V_{\text {int }}$ has crossed $V_{\text {REF }}$ and the comparator has toggled. The PG drives the transmitter, TX, which excites an external antenna coil at a resonant frequency of approximately 350 $\mathrm{MHz}$. The 2 pulses are then transferred wirelessly to a receiver unit where they are reconstructed by the receiver front-end. The conversion time of the reconstructed pulses, $\mathrm{T}_{\text {conv,rec }}$, can then be determined with the aid of a counter at the receiver end and the sensor current, $\mathrm{I}_{\mathrm{CELL}}$ can be derived by setting $\mathrm{t}_{2}$ equal to $\mathrm{T}_{\text {conv,rec }}$ in Eq. 1 . The simulated efficiency of thr PHM transmitter is $1.49 \mathrm{pJ} / \mathrm{bit}$.
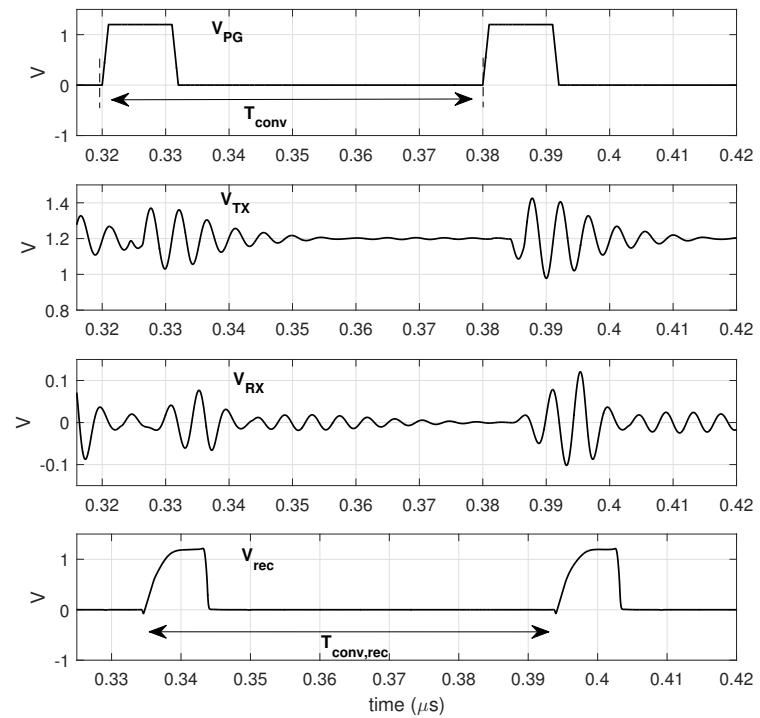

Fig. 10: Simulated transient operation of the wireless unit. Due to prohibitive simulation times the time scale of the wireless operation is much shorter than that of the dual-slope ADC.

\section{CONCLUSION}

This paper has presented the design of an energy-efficient 4-channel wireless potentiostat with reduced complexity, featuring a calibration strategy that does not require additional circuitry. Each channel can be operated independently and interfaced to disposable 3-terminal electrochemical sensors. The potentiostat features a novel wireless transmission architecture that can significantly reduce the energy consumption, which was simulated to be lower than $5 \mathrm{pJ} / \mathrm{bit}$ per wireless channel. The potentiostat chip lends itself to the development of handheld multi-sensor platforms for multiplexed PoC diagnostics.

\section{REFERENCES}

[1] Zarei, M., 2017. Portable biosensing devices for point-of-care diagnostics: Recent developments and applications. TrAC - Trends in Analytical Chemistry, 91, pp.26-41.

[2] Massicotte, G. et al., 2016. A CMOS Amperometric System for MultiNeurotransmitter Detection. IEEE Transactions on Biomedical Circuits and Systems, 10(3), pp.731-741.

[3] Li, H., Sam Boling, C. and Mason, A.J., 2016. CMOS Amperometric ADC with High Sensitivity, Dynamic Range and Power Efficiency for Air Quality Monitoring. IEEE Transactions on Biomedical Circuits and Systems, 10(4), pp.817-827.

[4] Schormans, M., Valente, V. and Demosthenous, A. Single-Pulse Harmonic Modulation for Short Range Biomedical Inductive Data Transfer. 2017 IEEE Biomedical Circuits and Systems Conference (BioCAS) Proceedings. 